\title{
Author Correction: Local generation of fumarate promotes DNA repair through inhibition of histone $\mathrm{H} 3$ demethylation
}

Yuhui Jiang, Xu Qian, Jianfeng Shen, Yugang Wang, Xinjian Li, Rui Liu, Yan Xia, Qianming Chen, Guang Peng,

Shiaw-Yih Lin and Zhimin Lu

Correction to: Nature Cell Biology https://doi.org/10.1038/ncb3209, published online 3 August 2015.

In the version of this Article originally published, the authors mistakenly used the same images for three different time points in Fig. $2 \mathrm{~d}$ : images for $24 \mathrm{~h}$ post-IR WT were also used for $0 \mathrm{~h}$ post-IR WT (top row, left panels) and for $1 \mathrm{~h}$ post-IR, T236A mutant (2nd row from the top, right panels). The correct images from these experiments have now been inserted in the figure in all online versions of the Article. In addition, results from this experiment and from two additional independent experiments have been uploaded to Figshare and the sentence 'Additional repeats of this experiment have been uploaded to Figshare ${ }^{28}$ ' has been added to the figure legend to highlight this fact. The Figshare doi has been added as reference 28. The new reference and corrected Fig. $2 \mathrm{~d}$ are shown below.

28. Jiang, Y. et al. FH pT236-gammaH2AX-1-2-3.tif. Figshare https://doi.org/10.6084/m9.figshare.5867907 (2018).

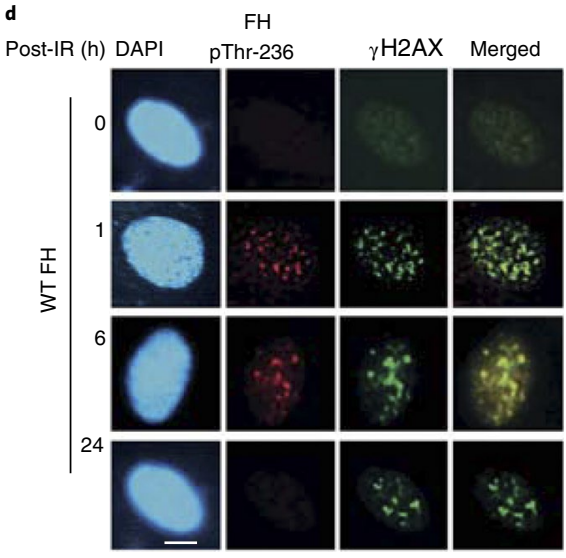

d

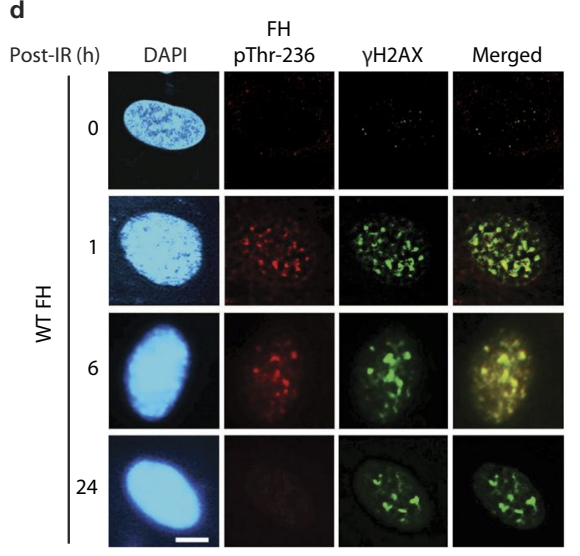

Original
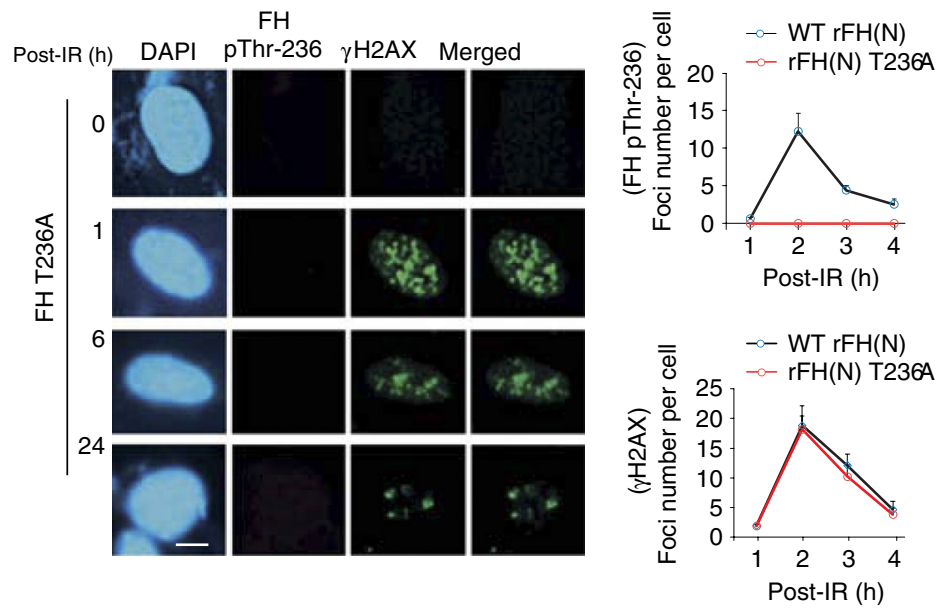

Corrected

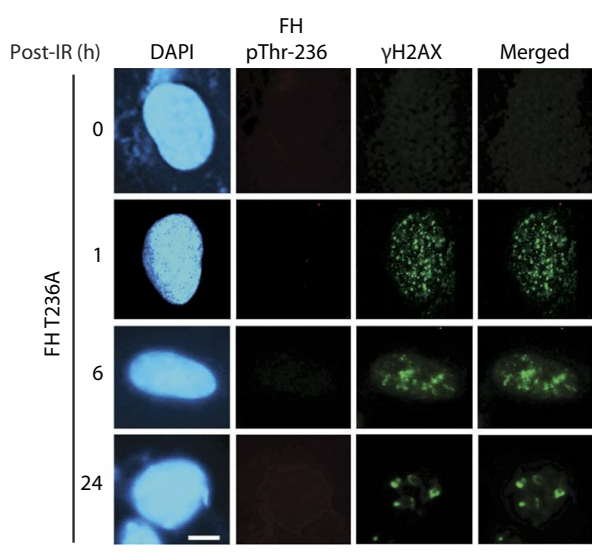

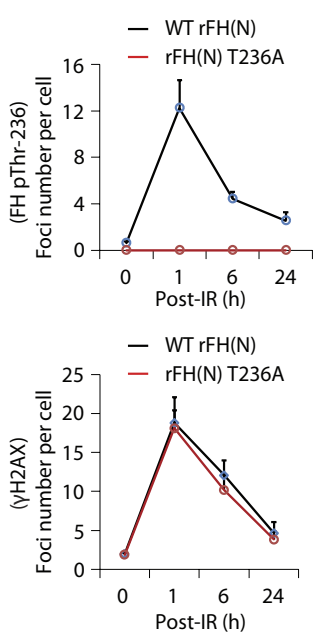

Fig. 2d | Original and corrected panels. 